\title{
Dual Laser Excitation of a Photochromic System: Application to DODCI
}

\author{
R. Duchowicz, * L. Scaffardi, R. E. Di Paolo, and J. O. Tocho \\ Centro de Investigaciones Opticas (CIOP). Casilla de Correo 124, 1900 La Plata. Argentina \\ (Received: April 23. 1991: In Final Form: August 29. 1991)
}

\begin{abstract}
A technique based on a combination of pump-and-probe fluorescence and absorption detection was developed in order to obtain the emission parameters of the photoisomeric (P) species of dyes with noncompletely overlapped absorption spectra of $P$ and the starting species $(N)$. Near total population transfer to the ground state of $P$ was achieved using a continuous wave high-nuence pump laser. A pulsed laser probed this population in its absorption region in such a way that the pulsed emission corresponded to fluorescence from $P$. Absorption measurements allowed us to follow the $P$ population and to establish its relationship with pump fluence and wavelength. The direct monitoring of the $\mathbf{P}$ concentration increased the accuracy of the previously reported values for several photophysical parameters. Temperature-dependent fluorescence quantum yield and lifetime, emission spectrum, and Arrhenius parameters of the nonradiative decay processes of $P$ from 3,3'-diethyloxadicarbocyanine iodide (DODCI) are given. The mechanism for the direct transformation between $N$ and $P$ through the singlet excited states of DODCl is re-evaluated.
\end{abstract}

\section{Introduction}

Photoisomerization processes of cyanine dyes have been analyzed through several techniques: flash photolysis, ${ }^{1,2}$ picosecond spectroscopy, ${ }^{3}$ photoacoustics, ${ }^{4}$ thermal grating. ${ }^{5}$ laser saturated absorption ${ }^{4}$ and fluorescence, ${ }^{7}$ absorption and emission spectroscopy with conventional sources, etc. These techniques exhibit a common drawback in the determination of the kinetic parameters of the photoisomeric species $P$ in an indirect way, using a photoisomerization model for the simultaneous fit of several parameters. This procedure leads to an inherent lack of precision. While

(1) Dempster, D. N.: Morrow, T.; Rankin, R.; Thompson. G. F. J. Chem Soc., Faraday Trans. 2 1972, 68, 1479.

(2) Rulliere. C. Chem. Phys. Lett. 1976, 46, 303

(3) Sibbett, W.; Taylor, J. R.; Welford, D. IEEE J. Quantum Electron. 1981. QEI7, 500

(4) Bilmes, G. L.; Tocho. J. O.: Braslavsky, S. E. Chem. Phys. Lett. 1987. 134. 335 .

(5) Zhu, X. R.; Harris, J. M. Chem. Phys. 1988, 124, 321

(6) Bãumler, W.; Penzkofer, A. Chem. Phys. 1990, 142, 431

(7) Scaffardi. L.; Bilmes, G. M.: Schinca, D.: Tocho. J. O. Chem. Phys. Lett. 1987, 140,163.

(8) Bāumier, W.; Penzkofer, A. Chem. Phys. 1990, 140,75 and references therein. in.

$0022-3644 / 92 / 2096-2501 \$ 03.00 / 0$ it can be used for either totally overlapped absorption-emission spectra (DTDCI, merocyanine 540, etc.) or partially overlapped spectra (DODCI. HITCI, etc.), it is only with using time-resolved picosecond spectroscopy that the different contributions can be directly obtained.

Some spectroscopic properties of $\mathbf{P}$ in photoisomerizable dyes with partially overlapped spectra have been reported previously. 9.10 These experiments were based on continuous wave (CW) and pulsed excitation of the sample. The $C W$ laser, tuned at the absorption wavelength of the normal $(N)$ ground-state species, produced a stationary population of the $\mathrm{P}$ ground state while the second laser (pulsed) probed this population. The pulsed fluorescence arose from $P$ free from interference from the $N$ emission.

In the present work, the measurements were performed at high pump fluence for which the photoequilibrium populations of all levels involved are shown to be independent of this pump fluence. Under this condition (saturation), the analysis of the results is

(9) Duchowicz. R.; Scaffardi, L.: Tocho, J. O. Chem. Phys. Lett. 1990, $170,497$.

(10) Bilmes. G. M.; Tocho, J. O.; Braslavsky. S. E. J. Phys. Chem. 1988, 92. 5958 .

(C) 1992. American Chemical Sncietv 


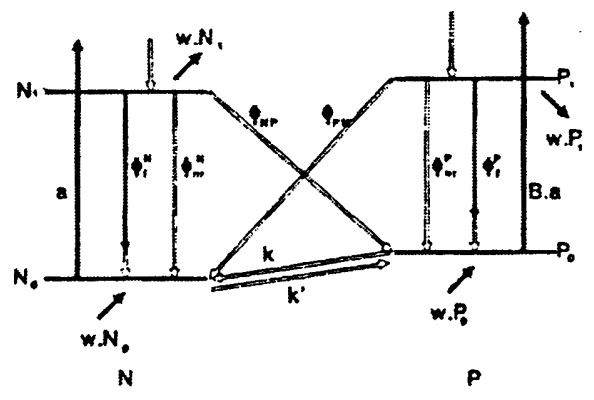

Figure 1. Four-level scheme used for calculation. Nonradiative transitions are indicated with empty lines.

greatly simplified and more reliable. Another modification is the use of a third laser beam as a second probe for the measurement of the $\mathrm{P}$ ground-state concentration $\left(\mathrm{P}_{0}\right)$ by absorption. Emission of the photoisomer was studied by detection of the fluorescence induced by the pulsed laser as a function of temperature.

This method was applied to DODCI, which has been extensively studied for its importance in pico- and femtosecond pulse generation." Although there is no final evidence about the structure of both isomers of DODCI, it is accepted that a cis-trans isomerization occurs during the transformation $\mathbf{N} \leftrightarrow \mathbf{P}$. A four-level diagram often is used to study the dynamics of DODCl. This model is of general application and independent of the precise conformation of the $\mathrm{N}$ and $\mathrm{P}$ isomers.

Figure 1 shows such a diagram where all possible intra- and interisomer transformations are indicated. The notation is given in the Appendix with the corresponding rate equations. When the photochromic system is in equilibrium, the fluence-dependent ground-state population can be expressed ( $\propto$ A2 of the Appendix) as

$$
\frac{P_{0}}{N}=\frac{\Phi_{\mathrm{NP} a}+k^{\prime}}{\left(\Phi_{\mathrm{NP}}+\mathrm{B} \Phi_{\mathrm{PN}}\right) a+k+k^{\prime}+w}
$$

For high intensity, $\sigma \Phi_{N P} \gg k+k^{\prime}+w, \propto q 1$ can be reduced to

$$
\frac{P_{0}}{N}=\frac{1}{1+p B}
$$

where $N$ is the total population, $p=\Phi_{\mathrm{PN}} / \Phi_{\mathrm{NP}}$, and $B=\sigma_{\mathrm{P}} / \sigma_{\mathrm{N}}$. Under this condition, the maximum population of $P$ depends on the excitation wavelength, as a result of the dependence of $B$ with this parameter, and is independent of the laser fluence.

\section{Materials and Methods}

3,3'-Diethyloxadicarbocyanine iodide (DODCI), laser grade, Eastman Kodak, in aerated solutions of analytical grade ethanol at low concentrations $\left((0.5-3) \times 10^{-6} \mathrm{M}\right)$ were employed in order to avoid dimerization and inner filter effects and to insure a homogeneous concentration of all species along the cell. The solutions were flowed through a standard fluorescence cuvette (1 $\times 1 \times 4 \mathrm{~cm}^{3}$ ) from a reservoir in a thermostatic bath to control the temperature within $0.2^{\circ} \mathrm{C}$. The rate of sample renewal by circulation, $w$, is related to the flow velocity in the cell, $v$, and the diameter of the irradiated zone, $d ; w=v / d \approx 10^{3} \mathrm{~s}^{-1}$ was estimated. Temperatures were measured in the cell by a $\mathrm{Cu}$-constantan thermocouple.

Fluorescence and absorption measurements were simultaneously performed with the setup in Figure 2. For the former, an $\mathrm{Ar}$ ion-pumped dye laser (Rhodamine 6G) or the Ar ion laser were focused on the cell. Simultaneously, a Rhodamine 640 flashlamp-pumped dye laser (Chromatix CMX-4, pulse width $=1 \mu \mathrm{s}$, peak energy $=1 \mathrm{~mJ}$, repetition rate $=5 \mathrm{~Hz}$ ) was aligned in the opposite direction with respect to the $\mathrm{CW}$ laser and focused with the lens, $L_{2}$, on the same spatial region of the cell. The pulsed fluorescence was detected perpendicularly to the excitation di-

(11) Fork, R. L.: Brito Cruz. C. H.: Becker, P. C.; Shank, C. V. Opt. Lett 1987, 12, 483.

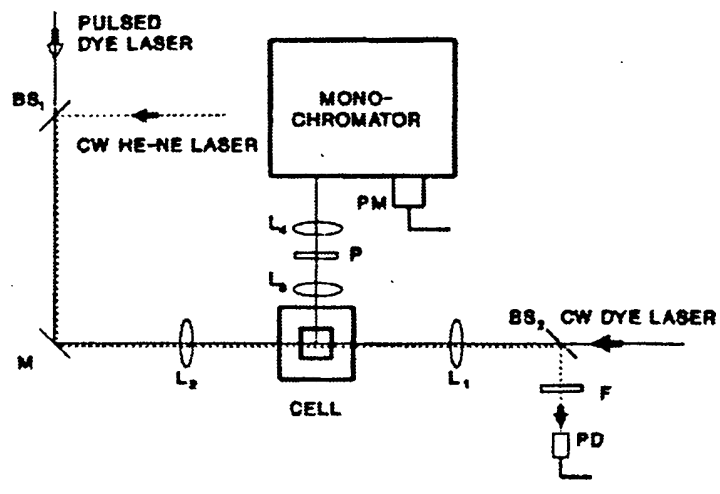

Figure 2. Experimental setup. Fluorescence excitation-detection path is shown by the continuous line, and the absorption path by the dashed line. $L_{1}, L_{2}, L_{3}$, and $L_{4}$ are focusing lenses; $P$ is a polarizer, $P M$ is a photomultiplier; $\mathrm{BS}_{1}$ and $\mathrm{BS}_{2}$ are beam splitters; $\mathrm{PD}$ is a photodiode; and $F$ is a filter (Schott OG590).

rection. Pulsed and $\mathrm{CW}$ laser beam polarizations were perpendicular to the plane defined by the incidence and observation directions. Fluorescence was focused through a system of lenses, $L_{3}$ and $L_{4}$, on the slit of the Ebert-mount scanning spectrometer (Jarrell Ash 82-025). The fluorescence spectrum was obtained by scanning the emission wavelength. A polarizer oriented at the magic angle 12 was placed between the lenses to eliminate fuorescence contributions arising from temperature-dependent rotational orientation effects. For spectrum correction, a second polarizer, side by side to the former but with vertical transmission. was used, and correction was carried out as in ref 7. Emission was detected using a Hammamatsu R-466 multialkali photomultiplier.

For the absorption measurements, a low-power CW He:Ne laser $(632.8 \mathrm{~nm})$ was used instead of the pulsed dye laser (dashed line in Figure 2). A photodiode (Spectra Physies Model 404) measured the He:Ne laser intensity transmitted through the cell with $\left(I_{1}\right)$ and without $\left(I_{2}\right)$ pump beam excitation. The absorption coefficient calculated as $\alpha_{\mathrm{p}}=\ln \left(I_{2} / I_{1}\right)$ is related to $P_{0}$ by

$$
\alpha_{\mathrm{p}}=\sigma_{\mathrm{p}}(632.8 \mathrm{~nm}) P_{0} l
$$

where $l$ is the length of the cell. It was assumed that the intensity absorbed by the normal species is negligible at this wavelength $\left(\sigma_{\mathrm{P}} \gg \sigma_{\mathrm{N}}\right)$. Both fluorescence and absorption signals were processed by a Boxcar integrator (EG\&G PAR 162-163) and plotted on an $Y-t$ recorder.

\section{Results and Discussion}

I. Po Population under a Stationary Saturated Regime. Saturation can be defined as the condition for which the absorption of $P$ is independent of the pump fluence. Under this condition, $\alpha_{p}$ was measured at various pump wayelengths in order to obtain the wavelength dependence of $P_{0}$ at photoequilibrium. $\alpha_{p}$ 's values normalized to the value obtainod with the pump at the isosbestic point, $B=1\left(\lambda_{a}=600 \mathrm{~nm}\right.$ for DODCl), are shown in Figure 3 . Eqs 2 and 3 can be combined to fit experimental values as

$$
\frac{\alpha_{\mathrm{P}}(\lambda)}{\alpha_{\mathrm{p}}(\lambda \alpha)}=\frac{P_{0}(\lambda)}{P_{0}\left(\lambda_{\alpha}\right)}=\frac{1+p}{1+p B}
$$

The best fit was obtained for $p=\Phi_{\mathrm{PN}} / \Phi_{\mathrm{NP}}=0.36 \pm 0.06$. $B$ was calculated from $\sigma$ values from ref 10 . The good fit of all dats supports the independence of $p$ with the pump wavelength. The value obtained for $p$ allows us to obtain the population of $P$ by using $\propto q 2$. From Figure 3 it can be seen that at saturation almost all molecules are converted from $N$ to $P$ for $B<1(\lambda<580 \mathrm{~nm})$, while for $B>1(\lambda>600 \mathrm{~nm})$ back-photoisomerization is an effective channel to return from $P$ to $N$.

II. Fluorescence Emission under Stationary Saturated Condltions. Il.1. P, Fhrorescence Emission Spectrum. By continuous

(12) Lessing, H. E.; v. Jena. A. Chem. Phys. Letr. 1976, 42, 213 
Dual Laser Excitation of a Photochromic System

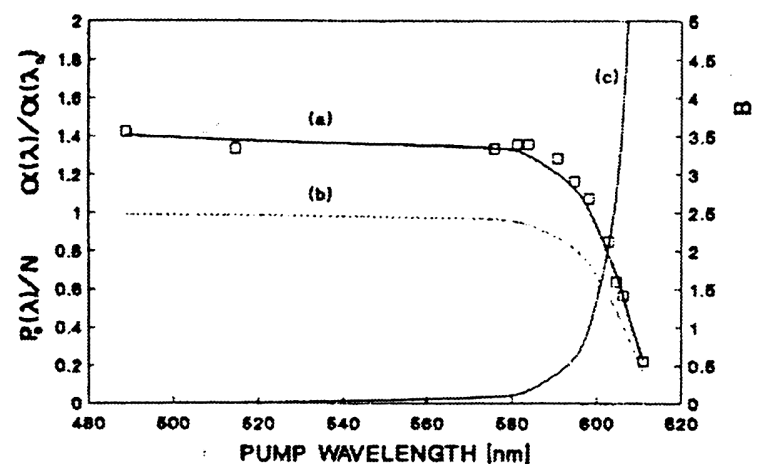

Figure 3. (a) Squares are experimental values of $\alpha(\lambda) / \alpha\left(\lambda_{\alpha}\right)$, a roomtemperature absorption ratio obtained from transmition measurements as a function of the excitation wavelength; the solid line corresponds to fitting values calculated using eq 4 (see text). (b) The dashed line represents the ratio of the normalized photoisomer ground-state population to the total population, $P_{0}(\lambda) / N$ (calculated from oq 2). (c) $B=$ $\sigma_{\mathrm{P}} / \sigma_{\mathrm{N}}$ with values from ref 10.

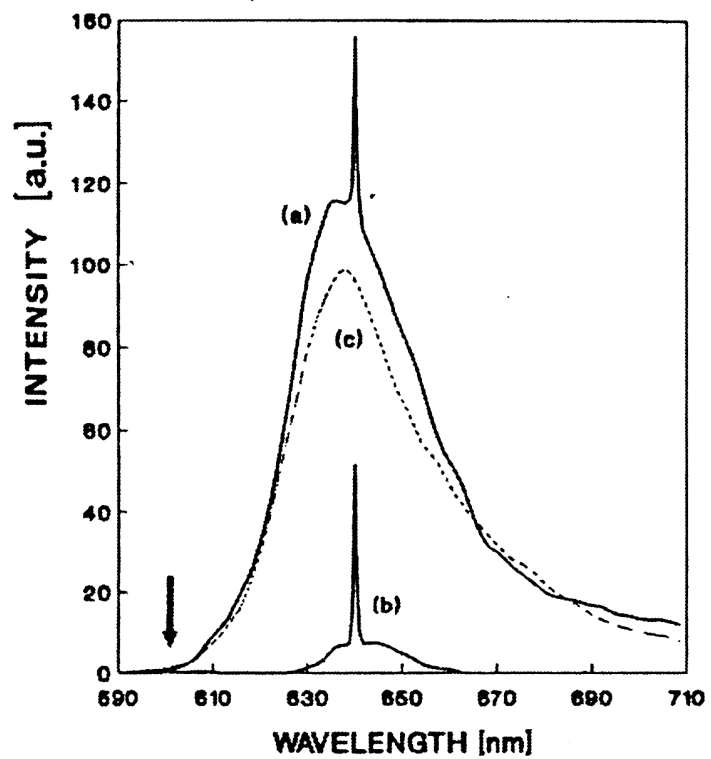

Figure 4. Pulsed fluorescent emission arising from $P_{1}$ obtained with (a) the double-excitation method (sce text) and (b) the cw pump off. (c) The dashed line is the corrected spectrum. The arrow indicates the position of the emission maximum of the normal species. Spikes are artifacts produced by scattering of the pulsed probe.

excitation of the sample with high fluence $\left(I_{0}=300 \mathrm{~W} / \mathrm{cm}^{2}\right)$ at $580 \mathrm{~nm}$, nearly all molecules are transferred to $P_{0}$ at room temperature. For pulsed excitation at $640 \mathrm{~nm}$, since $\sigma_{\mathrm{P}} P_{0} \gg \sigma_{\mathrm{N}} N_{0}$. a pulsed population in $\mathbf{P}$ is produced.

Figure 4 shows the pulsed fluorescent signal achieved with this double-excitation technique. The lower curve corresponds to the signal obtained with the $C W$ dye laser off. The arrow indicates the position of the emission maximum of the normal species, whose intensity level is found in this case to be lower than the pulsed noise. The CW signal, arising from $N_{1}$ fluorescence when the pump is on, is filtered by the low-impedance $(50 \Omega)$ detection system. The spike produced by scattering of the pulsed probe beam was subtracted using pure solvent in the cell in order to correct the spectrum.

II.2. Room-Temperature $P_{1}$ Fuorescence Parameters. With the absorption spectrum of the $P$ isomer from ref 10 and the Strickler and Berg formula, ${ }^{13}$ the radiative lifetime of the isomeric form was calculated as $P_{0}=4.0 \mathrm{~ns}$.

In order to obtain the nuorescence quantum yield of $P$, the emission from the photoisomeric form of DODCI was compared

(13) Strickler, S. J.; Berg, R. A. J. Chem. Phys. 1962, 37, 814.
The Journal of Physical Chemistry, Vol. 96. No. 6, 19922503

(peak emission, area under spectrum) with the normal species emission of 3,3'-diethylthiadicarbocyanine iodide (DTDCI) selocted for its similar absorption-emission properties. The sample was changed to the new dye, the cw pump was turned off, and the same low-energy pulsed excitation was used. An emission ratio of 0.254 was obtained. The absolute value for the fluorescence quantum yield of $P$ was calculated as $\Phi_{1}^{P}(D O D C I)=0.09 \pm 0.02$ using $\Phi_{1}^{N}(D T D C I)=0.36 \pm 0.05 .1^{4} \Phi_{\text {P }}^{P}$ results were higher than the value $0.06 \pm 0.02$ given in ref 10 , but they are within the experimental error

II.3. Analysis of Direct $P_{1} \rightarrow N_{1}$ Conversions. The possibility of the direct $P_{1} \rightarrow N_{1}$ conversion ${ }^{8}, 15$ can be discussed by use the spectrum of Figure 4 . The $N_{1}$ population can be analyzed in terms of the following processes: direct absorption by $\mathrm{N}_{0}$ or the $\mathrm{ab}$ sorption by $P_{0}$ followed by the passage from $P_{1}$ to $N_{1}$. The rate of population transferred in the latter case results in a maximum. This fact is due to both the low $\mathrm{N}_{0}$ population and the small absorption cross section of the normal species at the pulsed-probe wavelength. In other words, $N_{1}$ is only populated through the $P_{1} \Rightarrow N_{1}$ passage. In this case

$$
\frac{\mathrm{d} N_{1}}{\mathrm{~d} t}=k_{\mathrm{PN}}^{1} P_{1}-\frac{N_{1}}{\tau_{\mathrm{N}}} \simeq 0 \rightarrow N_{1}=k_{\mathrm{PN}} \tau_{N} P_{1}
$$

where stationary conditions for $N_{1}$ and $P_{1}$ are valid, since the pulse duration is much longer than the excited-state lifetimes. The fluorescence emission ratio between the $N_{1}$ and the $P_{1}$ emissions is estimated from

$$
\frac{I\left(\lambda_{N}\right)}{I\left(\lambda_{P}\right)}=\frac{\frac{N_{1} E\left(\lambda_{N}\right)}{\tau_{N} \lambda_{N}}}{\frac{P_{1} E\left(\lambda_{P}\right)}{\tau_{P} \lambda_{P}}} \simeq \Phi_{P N} \frac{\Phi_{\mathrm{N}}^{N}}{\Phi_{P}^{P}} \rightarrow \Phi_{P_{N}} \simeq \frac{I\left(\lambda_{N}\right)}{I\left(\lambda_{P}\right)} \frac{\Phi_{f}^{P}}{\Phi_{F}^{N}}
$$

where $\lambda_{N}$ and $\lambda_{P}$ correspond to the maximum emission of each species and $E(\lambda)$ is the line shape of the emissions, which was assumed equal for both species. Taking the intensities ratio as 0.01 and efficiencies ratio as $0.225\left(\Phi_{f}^{\mathrm{N}}=0.4^{2}\right.$ and $\Phi_{l}^{\mathrm{P}}=0.09$ obtained in section II.3), $\Phi_{\mathrm{PN}}^{1} \leq 2.25 \times 102$ results, a value that can be neglected in relation to $\Phi_{\mathrm{PN}}$.

The direct process $N_{1} \rightarrow P_{1}$ is more difficult to estimate. An overestimated limit can be obtained assuming a two-valley model for the upper potential energy surface of DODCI and an activated process where the depletion of population through the twisted state is neglected. In this case

$$
k_{\mathrm{NP}}=k_{\mathrm{N}} \exp \left(\Delta E / k_{\mathrm{B}} T\right)
$$

where $\Delta E=E_{\mathrm{N}}^{1}-E_{\mathrm{P}}^{1}$ is the energy difference between the $\mathrm{N}_{1}$ and $\mathrm{P}_{1}$ levels, $k_{\mathrm{B}}$ is the Boltzmann constant, and $T$ is the temperature. The highest value of $k_{\mathrm{NP}}^{1}$ is obtained using an energy value of $\Delta E$ $=770 \mathrm{cal} / \mathrm{mol}$, between the $\mathrm{N}_{0}$ and $\mathrm{P}_{0}$ levels reported. At room temperature, $k_{\mathrm{NP}}^{1}$ $\leq 9 \times 10^{-3} \mathrm{~ns}^{-1}$ and $\Phi \mathrm{NP}_{\mathrm{NP}} \leq 2 \times 10^{-2}$ result. Considering also the decay through the twisted state, these values should be much lower. $P_{1} \rightarrow N_{1}$ and $N_{1} \rightarrow P_{1}$ steps can be ruled out, as a first approach, from the model.

II.4. Temperature Dependence of Fluorescence Quantum Yield and Lifetime. When a $P_{0}$ equilibrium population is reached by cw pump at a given temperature, the amplitude of the pulsed fluorescence signal, $I(T)$, can be taken proportional to both the $P_{0}$ population and $\Phi_{f}^{P}(T)$. $P_{0}$ was measured as described before, between 0 and $55^{\circ} \mathrm{C}$; relative values referred to the room-temperature value are given in Figure 5 . The fluorescence intensity produced by the pulsed probe at $640 \mathrm{~nm}$ was measured in the same temperature range. $\Phi_{\mathrm{f}}^{\mathrm{P}}$ was calculated for each temperature as

$$
\Phi_{P}^{P}(T)=\frac{I(T)}{I\left(T_{0}\right)} \frac{P_{0}\left(T_{0}\right)}{P_{0}(T)} \Phi P\left(T_{0}\right)
$$

(14) Bilmes, G. M.; Tocho, J. O.; Braslavsky. S. E. J. Phys. Chem. 1989, 93. 6696.

(15) Arthurs, E. G.; Bradley, D. J.; Roddie, A. G. Chem. Phys. Lett. 1973, 


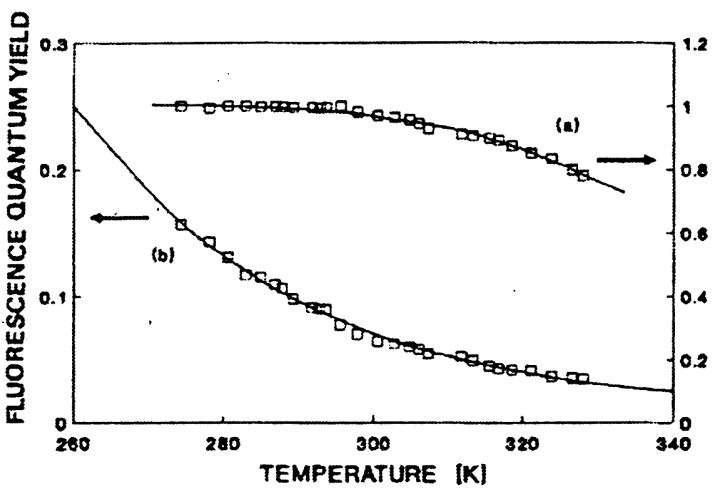

Figure 5. Squares are experimental points of (a) temperature dependence of the $\mathrm{P}$ population normalized at room temperature $\left(P_{0}(T) / P_{0}\left(T_{0}\right)=\right.$ $\alpha_{p}(T) / \alpha_{p}\left(T_{0}\right)$ was used from eq 3) and (b) temperature dependence of photoisomer fluorescence quantum yield $\left(\Phi_{P}^{P}(T)\right)$ from eq 5 . Solid lines are fittings using $\propto \mathrm{q} 1$ with $a=3 \times 10^{4} \mathrm{~ns}^{2 n}, w=3 \times 10^{-7}-\mathrm{ns}^{-1}$, and isomerization constants from refs 9 and 16 as parameters.

The values obtained from 0 to $55^{\circ} \mathrm{C}$ are also given in Figure 5. A small departure from saturation can be observed at high temperatures $\left(20 \%\right.$ at $\left.50^{\circ} \mathrm{C}\right)$. This fact is due to the increasing value of $k\left(P_{0} \rightarrow \mathrm{N}_{0}\right.$ transformation rate constant) with temperature.

Using $\Phi_{I}^{P}(T)$ and the radiative lifetime value obtained in section II.1, the lifetime of $\mathbf{P}_{1}$ for each temperature can be calculated from

$$
\tau_{P}(T)=\tau_{0}^{P} \Phi P(T)
$$

From $\tau_{0}^{p}$ and $\tau_{\mathrm{p}}$, the temperature dependence of the nonradiative processes can be analyzed as follow:

$$
k_{\mathrm{nr}}^{\mathrm{p}}=1 / \tau_{\mathrm{p}}-1 / \tau_{0}^{p}
$$

The results are plotted in the usual fashion (Arrhenius plot) in Figure 6a. The linearity of the plot indicates that only one radiationless process is involved in this temperature range. The activation energy was evaluated as $E_{\alpha}^{p}=5740 \pm 100 \mathrm{cal} \mathrm{mol}^{-1}$. This value turns out to be similar to those reported for the activation energies of the nonradiative processes from $\mathrm{N}_{1}$, the isomerization $\left(k_{\mathrm{NP}}\right)^{16}$ and the back-isomerization $\left(k_{\mathrm{PN}}\right){ }^{9}$. No contributions from $T$-independent nonradiative processes were found.

The nonradiative rate constant can be written as

$$
k_{\mathrm{nt}}^{\mathrm{P}}=F(\eta) \exp \left(-E_{0}^{\mathrm{P}} / R T\right)
$$

where $F(\eta)$ is a universal function of viscosity and $E_{0}^{p}$ is the intrinsic molecular barrier height, ${ }^{16}$ which is related only with molecular properties. By using different solvents (methanol, methanol-water, ethanol, and 2-propanol), an Arrhenius plot at constant viscosity can be constructed in order to obtain $E_{0}$. The results using $\eta=0.9 \mathrm{cP}$ and temperature-dependent viscosity value from ref 17 are shown in Figure 6b. An intrinsic barrier $E_{0}^{p}=$ $2830 \pm 200 \mathrm{cal} \mathrm{mol}^{-1}$ was obtained. This result is similar to that obtained for the $N_{1}$ nonradiative decay. ${ }^{16}$

\section{Conclusions}

Photophysical parameters for the DODCI P species have been obtained using a combination of pump-and-probe fluorescence and absorption methods. Fluorescence quantum efficiencies $\left(\Phi_{f}^{P}\right)$, radiative lifetime $\left(\tau_{0}^{P}\right)$, and lifetime $\left(\tau^{P}\right)$ in the range from 0 to $55^{\circ} \mathrm{C}$ were obtained with more accuracy than previously reported. ${ }^{1.1618}$ Analysis of the direct exchange between the excited singlet states indicates that the efficiencies for these processes are

(16) Velsko, S, P. Fleming, G. R Chem. Phys, 1982, $65,59$.

(17) Handbook of Chemistry and Physics: 62nd ed.; CRC Press: Boca Raton. FL, 1981-1982.

(18) Mialocq. J. C.; Boyd. A. W,; Jaraudias, J.: Sutton, J. Chem. Phys. Leti. 1976, 37, 236.

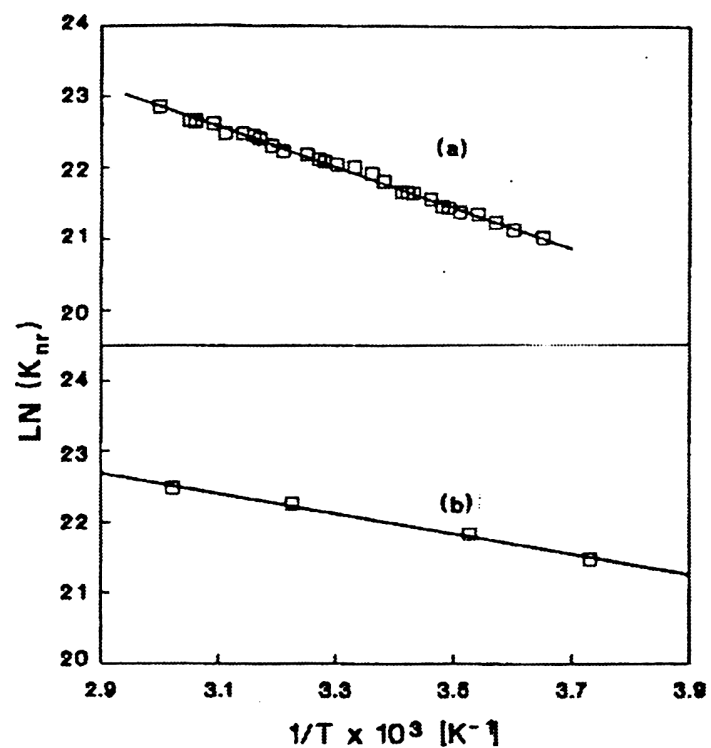

Figure 6. Arrhenius plots of photoisomer nonradiative rate constants in different solvents: (a) ethanol; (b) isoviscosity solutions $(\eta=0.9 \mathrm{cP})$ (methanol, methanol-water, ethanol, and 2-propanol were used).

very low. Therefore, they should be ruled out from the DODCI photoisomerization model.

The ratio between both isomerization quantum yields, $p=$ $\Phi_{\mathrm{PN}} / \Phi_{\mathrm{NP}}$, was found independent of pump wavelength. This fact is in disagreement with the results of Bāumler and Penzkofer. ${ }^{6}$ These authors found $p$ values of 1.35 at short pump wavelengths of 0.25 at the longest wavelength, and of a maximum of 3.5 at $570 \mathrm{~nm}$ as a result of the dependence with the excitation wavelength for both transfer efficiencies. The reason for such discrepancies is not clear at the moment. Higher concentrations and a lower pump intensity were used in the above-mentioned paper with respect to those of the present work. With the concentration used in ref 6, inhomogeneous population densities should be present along the cell. This fact was not considered by the authors. In addition, the interpretation of the results obtained below saturation is more difficult and sensitive to the fitting procedure. Furthermore, Velsko et al. ${ }^{19}$ have shown that the direct isomerization rate, $\Phi_{N P}$, is independent of pump wavelength.

Working at saturation condition, since fitting is reduced to a minimum, the interpretation of the photoisomerization process is simplified and the results are more reliable. The technique developed can be extended to the 8 ther molecules with incomplete overlapping of the absorption spectra.

Acknowledgment. The work was supported by the Consejo Nacional de Investigaciones Cientificas y Técnicas of Argentina (CONICET) (Grant PID 3-060100/88). R.D., L.S., and J.O.T. are members of the Carrera del Investigador from CONICET and members of Departamento de Fisica of Universidad Nacional de La Plata. R.E.DiP. thanks the Comision de Investigaciones Cientificas de la Provincia de Buenose Aires (CICBA) for a fellowship.

\section{Appendix}

Equations for the Calculation of Populations under Photoequilibrium. The scheme of Figure 1 summarizes the four-level kinetic model used here to formulate the set of differential rate equations that leads to eq 1 . The direct passage between excited states $N_{1}$ and $P_{1}$ is ruled out in this analysis by taking into account

(19) Velsko, S. P.; Waldeck, D. H.: Fleming, G. R. J. Chem. Phys. 1983, 78.240 
the results of section II.3.

$$
\begin{aligned}
& \frac{\mathrm{d} N_{0}}{\mathrm{~d} t}= \\
& -(a+w) N_{0}+\frac{\left(1-\Phi_{\mathrm{NP}}\right)}{\tau_{\mathrm{N}}} N_{1}+\frac{\Phi_{\mathrm{PN}}}{\tau_{\mathrm{P}}} P_{1}+k P_{0}-k^{\prime} N_{0}+w N \\
& \frac{\mathrm{d} N_{1}}{\mathrm{~d} t}=a N_{0}-\left(\frac{1}{\tau_{\mathrm{N}}}+w\right) N_{1} \\
& \frac{\mathrm{d} P_{1}}{\mathrm{~d} t}=B a P_{0}-\left(\frac{1}{\tau_{\mathrm{P}}}+w\right) P_{1} \\
& \frac{\mathrm{d} P_{0}}{\mathrm{~d} t}=\frac{\Phi_{\mathrm{NP}}}{\tau_{\mathrm{N}}} N_{1}+\frac{\left(1-\Phi_{\mathrm{PN}}\right)}{\tau_{\mathrm{P}}} P_{1}-(B a+k+w) P_{0}+k^{\prime} N_{0}
\end{aligned}
$$

In $\propto \mathrm{A} A 1, N_{0}, N_{1}$ and $P_{0}, P_{1}$ are the ground- and excited-state populations of the normal and photoisomer species, respectively, and $N=N_{0}+N_{1}+P_{0}+P_{1} . B=\sigma_{\mathrm{P}} / \sigma_{\mathrm{N}}$, with $\sigma_{\mathrm{P}}$ and $\sigma_{\mathrm{N}}$ as the absorption cross section coefficients of the $\mathrm{P}$ and $\mathrm{N}$ species. a $=I_{1} \sigma_{N}$, which is the rate of light absorption, where $I_{1}$ is the excitation fluence. $w$ is the rate of sample renewal within the irradiated volume by circulation of the solution. $k$ and $k^{\prime}$ are the rate constants of the $P_{0} \rightarrow N_{0}$ and $N_{0} \rightarrow P_{0}$ processes, and $\tau_{N}$ and $\tau_{p}$ are the lifetimes of excited states for the normal and photoisomeric species. $\Phi_{\mathrm{NP}}$ and $\Phi_{\mathrm{PN}}$ are the quantum yields of $N_{1} \rightarrow P_{0}$ and $P_{1} \rightarrow N_{0}$ isomerization processes.

Under steady-state irradiation conditions and low absorption, the following expression can be obtained:

$$
\frac{P_{0}}{N}=\frac{\Phi_{\mathrm{NP}} a+k^{\prime}}{\left(\Phi_{\mathrm{NP}}+B \Phi_{\mathrm{PN}}\right) a+k+k^{\prime}+w}
$$

where excited-state populations are taken negligibly small because the pump intensity, $I_{l}$, is small compared to the saturation intensity for the excited-state populations, $I=h \nu / \sigma$ which are in the $\mathrm{MW} / \mathrm{cm}^{2}$ region and the depopulation of $N_{1}$ and $P_{1}$ by circulation $\left(w \ll 1 / \tau_{N} ; 1 / \tau_{P}\right)$ are neglected. 